\title{
Vessel Track Prediction Based on Fractional Gradient Recurrent Neural Network with Maneuvering Behavior Identification
}

\author{
Han Xue iD and Tian Chai $(i D$ \\ College of Navigation, Jimei University, Xiamen 361021, China \\ Correspondence should be addressed to Tian Chai; chaitian2006@163.com
}

Received 8 February 2021; Revised 20 February 2021; Accepted 4 March 2021; Published 13 March 2021

Academic Editor: Jiangbo Qian

Copyright (C) 2021 Han Xue and Tian Chai. This is an open access article distributed under the Creative Commons Attribution License, which permits unrestricted use, distribution, and reproduction in any medium, provided the original work is properly cited.

\begin{abstract}
To improve the accuracy of ship track prediction, a fractional-order gradient descent method is adopted into a recurrent neural network (RNN). The convergence of the proposed algorithm is proved. Identification of ship maneuvering behavior, atmospheric information, and oceanographic information is considered in vessel tack prediction. The ship track of Xiamen Port is predicted using the new algorithm. Error analysis is made with different factional orders and traffic busy degrees. Results show that the testing and training error differs with different fractional orders. The predicted track results can not only improve the efficiency of marine traffic management but also prevent and warn the safety accidents, so as to avoid accidents.
\end{abstract}

\section{Introduction}

Ship trajectory prediction is to predict the ship's future navigation position by analyzing the history and current trajectory law of the target ship under the premise of considering the external environment around the target ship and the performance of the ship's own equipment. Ship track prediction uses the current navigation state of the ship, combined with the operation of the pilot, the surrounding environment information and the ship's maneuverability, and other constraints to predict the future position of the ship. This is a basic technology of marine traffic management and a necessary condition to realize the automation and intelligence of marine traffic management. The predicted track results can not only improve the efficiency of marine traffic management but also prevent and warn the safety accidents, so as to avoid accidents.

A long short-term memory network (LSTM) deep learning model was proposed [1]. Vessel trajectory prediction using historical AIS data was presented [2]. Gaussian process model was studied [3].

The research of ship track prediction adopts a variety of advanced artificial intelligence optimization algorithms, such as neural network. In 1990, the first fully connected recurrent neural network (RNN), namely, Elman network, was proposed [4]. In 1997, a bidirectional RNN with deep structure was proposed [5]. In 1997, LSTM was proposed [6]. However, due to the problems of gradient vanishing and gradient exploding, RNN training is very difficult and its application is very limited. An adaptive optics prediction algorithm based on LSTM was proposed [7]. An adaptive PSO optimized LSTM network was proposed [8]. A modified atom search optimization-based deep RNN was proposed for epileptic seizure prediction [9].

However, gradient descent sometimes has some disadvantages, such as fluctuation. Therefore, its improvement such as the introduction of fractional-order calculus is needed. The variable-order fractional discrete-time neural network was proposed [10]. A fractional-order complex-valued RNN was designed [11]. A fractional-order memristive RNN was proposed [12].

In the intelligent maritime traffic system, some research achievements have been made. A ship trajectory and navigation state prediction method based on AIS data was proposed, with properly designed learning, motion modelling, and knowledge base-assisted particle filtering processes [13]. A density-based clustering and bidirectional long short-term memory- (BLSTM-) based supervised learning 
were proposed for vessel trajectory reconstruction [14]. Douglas Peucker (DP) and kernel density estimation (KDE) algorithms were used for the compression and visualization of large ship trajectory and the acceleration of GPU [15]. A continuous berth scheduling problem was solved with the objective of minimizing the total operating cost [16].

However, due to the problems of gradient vanishing and gradient exploding, RNN training is sometimes very difficult. This paper adopts fractional order. The influence of fractional order and learning rate on the algorithm performance is analyzed. The main contributions of this paper are as follows:

(1) Fractional-order calculus is applied to gradient descent for training recurrent neural network

(2) The convergence of fractional gradient recurrent neural network is proved

(3) Identification of ship maneuvering behavior, atmospheric information, and oceanographic information is considered in vessel tack prediction

The remainder of this paper can be divided into the following four parts. Section 2 mainly proposes the related work. The vessel track prediction based on fractional gradient recurrent neural network is then presented in Section 3. Extensive experiments are carried out in Section 4. We end this work by summing up the main contributions in Section 5 .

\section{Related Work}

2.1. Identification of Ship Maneuvering Behavior. There are three types of ship maneuvering: keeping current course, changing course, and changing speed. Sample three points. $P_{i}$ is the $i$-th trajectory point in the trajectory. The time interval between the adjacent points $P_{(i-1)}$ and $P_{(i+1)}$ is $\Delta t$. $V_{(i)}$ is the velocity from $P_{(i)}$ to $P_{(i-1)}$. $V_{(i+1)}$ is the vector velocity from $P_{(i)}$ to $P_{(i+1)}$ :

$$
\begin{gathered}
V_{(i)}=\frac{P_{(i)} P_{(i-1)}}{\Delta t}, \\
V_{(i+1)}=\frac{P_{(i)} P_{(i+1)}}{\Delta t} .
\end{gathered}
$$

$C$ represents the course of the ship and $V$ is the speed of the ship. $a_{\mathrm{s}}$ is the ship speed change rate and $a_{\mathrm{c}}$ is the course change rate:

$$
\begin{aligned}
& a_{s}=\frac{\Delta v}{\Delta t}=\frac{V_{(i+1)}-V_{(i)}}{\Delta t}, \\
& a_{c}=\frac{C_{(i+1)}-C_{(i)}}{\Delta t} .
\end{aligned}
$$

Four threshold parameters are set to divide the ship maneuvering behavior mode:

$$
\text { Threshold }=\left(a_{\text {Weak }}, a_{\text {Sharp }}, a_{\text {Acceleration }}, V_{\text {Zero }}\right),
$$

where $a_{\text {Weak }}$ is weak yaw with changing course, $a_{\text {Sharp }}$ is the strong yaw with changing course, $V_{\text {Zero }}$ is the speed threshold of berthing, and $a_{\text {Acceleration }}$ is the variable speed threshold.

The identification model of ship maneuvering mode is set according to different kinematic constraints. The identification rules are as follows:

(a) If $V_{(i)}<V_{\text {Zero }}$ and $V_{(i+1)}<V_{\text {Zero }}, P_{i}$ belongs to staying

(b) If $a_{s}<0$ and $\left|a_{s}\right|>a_{\text {Acceleration }}, P_{i}$ belongs to deceleration navigation behavior

(c) If $a_{s}>0$ and $\left|a_{s}\right|>a_{\text {Acceleration }}, P_{i}$ is considered to be accelerated

(d) When $V_{(i)}>V_{\text {Zero }}, V_{(i+1)}>V_{\text {Zero }},\left|a_{s}\right|<a_{\text {Acceleration }}$, and $\left|a_{c}\right|<a_{\text {Weak }}, P_{i}$ belongs to keeping direction and speed

(e) If $a_{c}<0$ and $a_{\text {Weak }}<\left|a_{c}\right|<a_{\text {Sharp }}, P_{i}$ belongs to weak yaw with left rudder

(f) If $a_{c}>0$ and $a_{\text {Weak }}<\left|a_{c}\right|<a_{\text {Sharp }}, P_{i}$ belongs to weak yaw with right rudder

(g) If $a_{c}<0$ and $\left|a_{c}\right|>a_{\text {Sharp }}, P_{i}$ belongs to strong yaw with left rudder

(h) If $a_{c}>0$ and $\left|a_{c}\right|>a_{\text {Sharp }}, P_{i}$ belongs to strong yaw with right rudder

2.2. Recurrent Neural Network. RNN establishes weighted connections between neurons in layers. With the continuous progress of the sequence, the former hidden layer will affect the later hidden layer. $o$ represents the output. $y$ represents the value given by the neurons, and $L$ represents the loss function. $x$ is the input vector. $w$ denotes the weight of the neural network. The loss function is accumulated with sequence. Since the weights are shared in RNN, weights in RNN are the same.

The error is defined as follows:

$$
e(t)=o(t)-y(t)
$$

The loss function is defined as follows:

$$
L(t)=\frac{1}{2} \sum_{k=1}^{m} e_{k}^{2}(t)
$$

Denote

$$
\begin{gathered}
\Delta w(t)=w(t+1)-w(t), \\
v_{k}(t)=\sum_{j=1}^{m} w_{k j}(t) y(t) .
\end{gathered}
$$

2.3. Fractional-Order Calculus. The Riemann-Liouville definition of fractional calculus is as follows.

Definition 1. For the absolute integrable function $x(t)$ in the interval $\left[t_{0}, t\right]$, its Riemann-Liouville integral is as follows: 


$$
{ }_{t_{0}} D_{t}^{\alpha} x(t)=\frac{1}{\Gamma(\alpha)} \int_{t_{0}}^{t}(t-\tau)^{\alpha-1} x(\tau) \mathrm{d} \tau,
$$

where $\alpha$ is the fractional order, whose real part is positive. $\Gamma(x)$ is the gamma function defined as follows:

$$
\Gamma(x)=\int_{0}^{\infty} e^{-t} t^{x-1} \mathrm{~d} t .
$$

Definition 2. For the absolute integrable function $x(t)$ in the interval $\left[t_{0}, t\right]$, its Riemann-Liouville differential is defined as follows:

$$
{ }_{t_{0}} D_{t}^{\alpha} x(t)=\frac{d^{m}}{d t^{n}}\left[\frac{1}{\Gamma(m-\alpha)} \int_{t_{0}}^{t}(t-\tau)^{m-\alpha-1} x(\tau) \mathrm{d} \tau\right],
$$

where $\alpha \in[m-1, m) . m$ is a positive integer.

Definition 3. For $f(x)=\left(x-x_{0}\right)^{v}$, the following formula holds:

$$
x_{0} D_{x}^{p} f(x)=\frac{\Gamma(v+1)}{\Gamma(v-p+1)}\left(x-x_{0}\right)^{v-p} .
$$

2.4. Vessel Track Prediction Based on Neural Network. The neural network model is used to learn the current navigation conditions and the driving habits of the ship drivers. The navigation state of the next moment is predicted by the historical speed, course, and meteorological and hydrological information. Thus, the navigation state of the ship in the future is predicted by recursive iteration.

The position of the ship at the next moment is predicted by the longitude and latitude, speed, course of the ship, and the interference of the current marine environment at the last moment. Take the historical dynamic navigation data and the historical sea wind and current data as the input of the neural network. Take the expected ship position as output, and the neural network is trained by comparing the difference between the real position and output position. The trained neural network can predict the position change of the ship through the dynamic data of the ship's current navigation and the interference of sea wind and current. Fractional-order training is adopted to further improve the prediction accuracy of the model.

The dynamic data of ship navigation, sea wind, and current interference are as follows:

$$
x(t)=\{V s, C s, V w, C w, H w, H t\},
$$

where $V s$ is the speed of the ship, $C s$ is the course of the ship, $V w$ is the speed of sea wind around the ship, $C w$ is the direction of the wind around the ship, $\mathrm{Hw}$ is the height of the wave around the ship, and $H t$ is the height of the tidal around the ship. The number of input layer nodes is 6 , and the number of output layer nodes is 2 . The hidden layer is defined as one layer. Figure 1 shows the track prediction method by neural network.

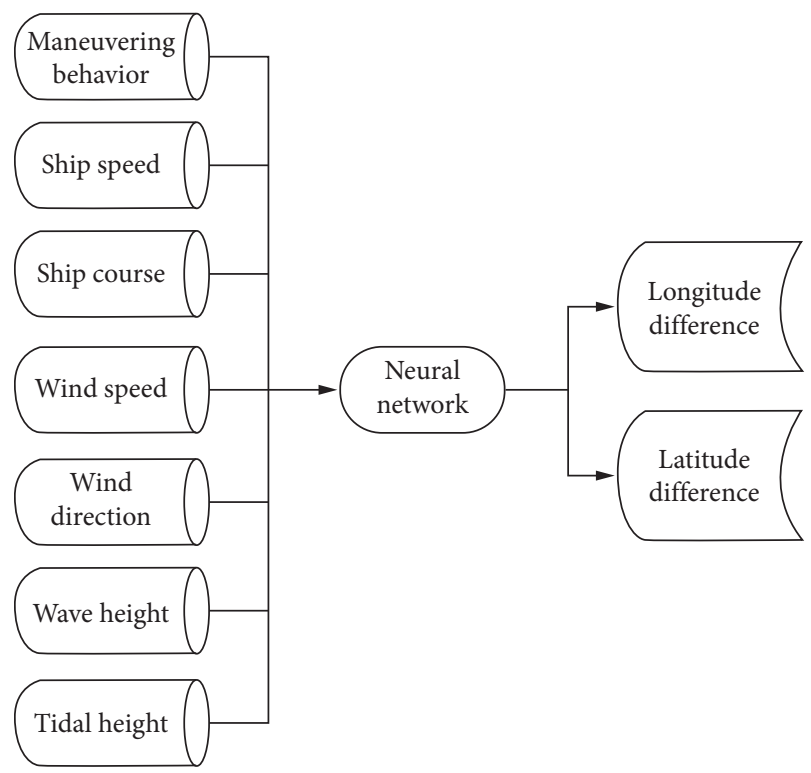

FIGURE 1: Track prediction method by neural network.

\section{Main Results}

3.1. FRNN Algorithm. The weight is updated using the gradient:

$$
w_{k j}(t+1)=w_{k j}(t)-\eta D_{w k j}^{\alpha} L(t) .
$$

The fractional-order derivative of $L(t)$ is as follows:

$$
D_{w k j}^{\alpha} L(t)=\frac{\partial L(t)}{\partial v_{k}(t)} \frac{w_{k j}^{1-\alpha}(t) y_{j}(t)}{\Gamma(2-\alpha)} .
$$

The derivative of $L(t)$ is as follows:

$$
\nabla_{w k j} L(t)=\frac{\partial L(t)}{\partial v_{k}(t)} y .
$$

Substituting (16) into the first item of (15) yields

$$
w_{k j}(t+1)=w_{k j}(t)-\eta \frac{\partial L(t)}{\partial v_{k}(t)} \frac{w_{k j}^{1-\alpha}(t) y_{j}(t)}{\Gamma(2-\alpha)} .
$$

Substituting (18) into the first item of (8) yields

$$
\Delta w_{k j}(t)=-\eta \frac{\partial L(t)}{\partial v_{k}(t)} \frac{w_{k j}^{1-\alpha}(t) y_{j}(t)}{\Gamma(2-\alpha)} .
$$

Substituting (19) into the first item of (17) yields

$$
\nabla_{w k j} L(t)=-\frac{\Gamma(2-\alpha)}{\eta} w_{k j}^{\alpha-1}(t) \Delta w_{k j}(t) .
$$

\subsection{Convergence of FRNN}

Lemma 1. The loss function $L$ is monotonically decreasing,

$$
L(w(t+1)) \leq L(w(t)) .
$$


Proof. Based on the Taylor mean value theorem,

$$
\Delta L=L(w(t+1))-L(w(t))=L^{\prime} \Delta w(t)+\frac{1}{2} L^{\prime \prime}\|\Delta w(t)\|^{2} .
$$

Applying (20) to (22), we have

$$
\Delta L<\left[-\frac{\Gamma(2-\alpha)}{\eta} w^{\alpha-1}(t)+c\right]\|\Delta w(t)\|^{2},
$$

where $c>1 / 2 L^{\prime \prime}$. From (23), one can obtain that (21) will hold if the following is satisfied:

$$
\eta \leq \frac{\Gamma(2-\alpha)\|w(t)\|^{\alpha-1}}{c} .
$$

Lemma 2. The set $\{L(t)\}$ is convergent,

$$
\lim _{t \longrightarrow \infty} L(w(t))=L^{*} \text {. }
$$

Proof. From (7), we have $L(w(t)) \geq 0$.

From Lemma 1, $L(w(t+1)) \leq L(w(t))$. Therefore, there exits $L^{*} \geq 0$ satisfying

$$
\lim _{t \rightarrow \infty} L(w t)=L^{*}
$$

Theorem 1 Assume (24) is valid. Then, the set $\left\{D_{w(t)}^{\alpha} L(w(t))\right\}$ converges to zero,

$$
\lim _{t \longrightarrow+\infty}\left\|D_{w(t)}^{\alpha} L(w(t))\right\|=0 .
$$

Proof. Denote

$$
\lambda=\frac{\Gamma(2-\alpha)}{\eta} w^{\alpha-1}(t)-c \geq 0 .
$$

From (23), one can obtain

$$
\begin{aligned}
& L(w(t+1)) \\
& \leq L(w(t))-\left[\frac{\Gamma(2-\alpha)}{\eta} w^{\alpha-1}(t)-c\right]\|\Delta w(t)\|^{2} \\
& =L(w(t))-\lambda\|\Delta w(t)\|^{2} \\
& =L(w(0))-\lambda \sum_{t=0}^{k}\|\Delta w(t)\|^{2} .
\end{aligned}
$$

Since $L(w(t)) \geq 0$, we have

$$
\lambda \sum_{t=0}^{k}\|\Delta w(t)\|^{2} \leq L(w(0)) .
$$

When $t \longrightarrow+\infty$,

$$
\sum_{t=0}^{\infty}\|\Delta w(t)\|^{2} \leq \infty
$$

Thus, we have

$$
\lim _{t \rightarrow \infty}\|\Delta w(t)\|=0
$$

Substituting (15) into (8) yields

$$
\Delta w(t)=-\eta D_{w(t)}^{\alpha} L(t) .
$$

Substituting (33) into (32) yields

$$
\lim _{t \longrightarrow \infty}\left\|-\eta D_{w(t)}^{\alpha} L(t)\right\|=0 \text {. }
$$

Thus, we have

$$
\lim _{t \longrightarrow+\infty}\left\|D_{w(t)}^{\alpha} L(w(t))\right\|=0 .
$$

From Theorem 1, we conclude that FRNN converges.

3.3. Trajectory Prediction Based on FRNN. The process of the FRNN algorithm for ship track prediction is shown as follows:

Step 1: initialize the neural network model

Step 2: input training samples

Step 3: calculate the hidden layer

Step 4: calculate the output layer

Step 5: calculate the output error

Step 6: if the learning requirement is satisfied, turn to step 2 until the training results meet the requirements

Step 7: initialize the neural network with the optimal weights

Step 8: input data needed for prediction into the trained neural network and output prediction results

\section{Results and Discussion}

4.1. Datasets. The AIS data used for prediction are the ship track data of Xiamen port. The original data should remove the wrong AIS data in the pretreatment. The records of obvious errors in AIS data include the following three types:

(1) The length of the ship's code is not 9 digits or unreasonable record

(2) The longitude and latitude of the ship exceed the reasonable range, for example, longitude $>180$ or latitude $>90$ or negative longitude and latitude

(3) The ship's speed and course exceed the reasonable range, for example, course $>360$ or speed $<0$

\subsection{Maneuvering Behavior Identification}

4.2.1. Constant Speed Mode. Table 1 lists information of MSC Bilbao ship, which belongs to constant speed mode.

The trajectory is plotted in Figure 2.

4.2.2. Acceleration Mode. Table 2 lists information of HAIYANG989 ship, which belongs to acceleration mode.

The trajectory is plotted in Figure 3. 
TABle 1: Constant speed of MSC Bilbao.

\begin{tabular}{lcccccc}
\hline Longitude & Latitude & $C$ & $V$ & Head & $a_{\mathrm{c}}$ & $a_{s}$ \\
\hline 122.198332 & 29.863167 & 334.0 & 12.7 & 335 & - & - \\
122.197835 & 29.864166 & 334.0 & 12.7 & 335 & 0 & 0 \\
122.197168 & 29.865333 & 334.0 & 12.7 & 335 & 0.98 & 0 \\
122.196501 & 29.8665 & 335.0 & 12.7 & 335 & 0 & 0 \\
122.195919 & 29.8675 & 335.0 & 12.7 & 335 & 0 & 0 \\
122.195337 & 29.8685 & 335.0 & 12.7 & 335 & - & - \\
\hline
\end{tabular}

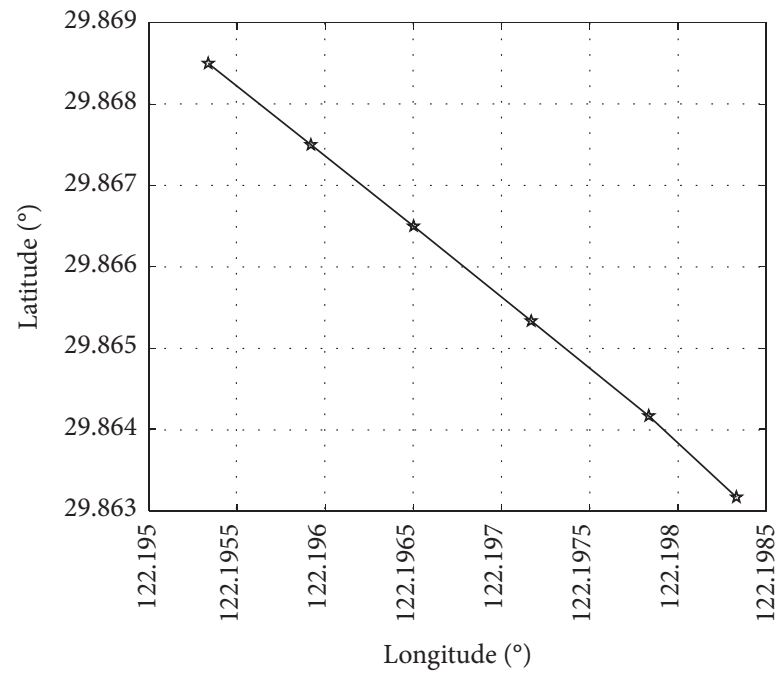

Figure 2: Track of MSC Bilbao.

TABle 2: Acceleration of HAIYANG989.

\begin{tabular}{lcccccc}
\hline Longitude & Latitude & $C$ & $V$ & Head & $a_{\mathrm{c}}$ & $a_{s}$ \\
\hline 122.015007 & 29.929411 & 126.7 & 8.3 & 152 & - & - \\
122.016439 & 29.928562 & 120.3 & 8.3 & 150 & -8 & 0 \\
122.019588 & 29.927161 & 112.3 & 8.3 & 148 & 2.9 & 0.3 \\
122.02207 & 29.926304 & 115.2 & 8.6 & 150 & -1.9 & 0 \\
122.022868 & 29.925993 & 113.3 & 8.6 & 150 & -4.1 & 0.1 \\
122.025456 & 29.925104 & 109.2 & 8.7 & 151 & 10 & 0.5 \\
122.030404 & 29.922681 & 119.2 & 9.2 & 153 & -4.3 & 0 \\
122.037272 & 29.921108 & 114.9 & 9.2 & 149 & - & - \\
\hline
\end{tabular}

4.2.3. Deceleration Mode. Table 3 lists information of SANTA LAURA ship, which belongs to deceleration mode.

The trajectory is plotted in Figure 4.

4.2.4. Turn Slightly to the Left. Table 4 lists information of SOFIE MAERSK ship, which belongs to turning slightly to the left mode.

The trajectory is plotted in Figure 5.

4.2.5. Turn Slightly to the Right. Table 5 lists information of HAIYANG989 ship, which belongs to turning slightly to the right mode.

The trajectory is plotted in Figure 6.

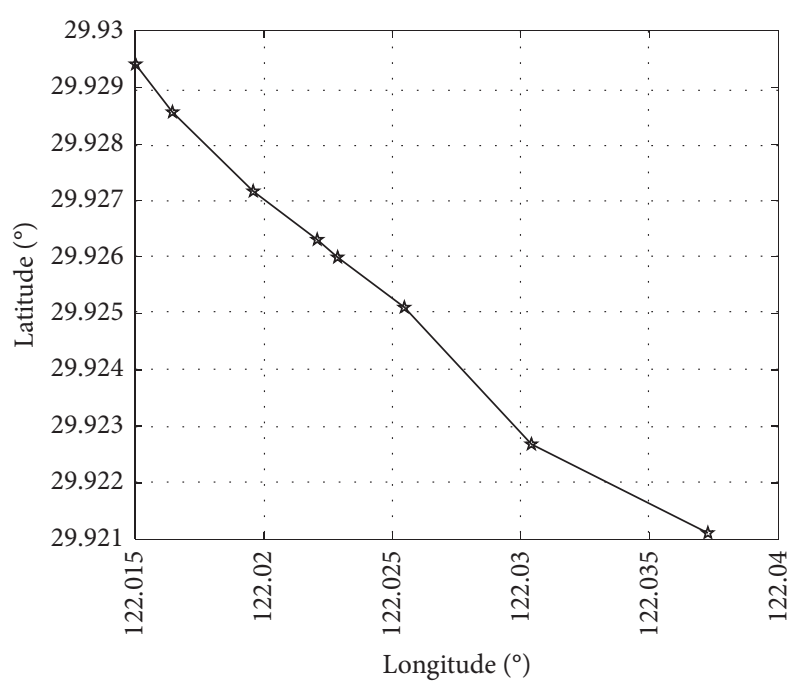

Figure 3: Track of HAIYANG989.

TABle 3: Deceleration of SANTA LAURA.

\begin{tabular}{lcccccc}
\hline Longitude & Latitude & $C$ & $V$ & Head & $a_{c}$ & $a_{s}$ \\
\hline 122.190856 & 29.87328 & 331 & 12.9 & 333 & - & - \\
122.189895 & 29.874767 & 333 & 12.9 & 333 & 3.0 & -0.9 \\
122.189412 & 29.875981 & 334 & 12.6 & 333 & 0.8 & -0.3 \\
122.188301 & 29.878043 & 334.8 & 12.3 & 333 & 2.3 & -0.9 \\
122.187191 & 29.880105 & 335.5 & 12 & 333 & 0.9 & -1.2 \\
122.186174 & 29.88207 & 335.8 & 11.6 & 333 & 0.3 & -0.2 \\
122.185677 & 29.883038 & 336.1 & 11.4 & 332 & -0.6 & -0.6 \\
122.184937 & 29.884462 & 335.9 & 11.2 & 332 & -0.3 & -0.3 \\
122.184692 & 29.884932 & 335.8 & 11.1 & 332 & -1.1 & -0.1 \\
122.184183 & 29.885851 & 334.8 & 11 & 332 & -3.2 & -0.3 \\
122.183675 & 29.886768 & 333.7 & 10.9 & 332 & -0.9 & -0.6 \\
122.183154 & 29.887667 & 333.4 & 10.7 & 333 & - & - \\
\hline
\end{tabular}

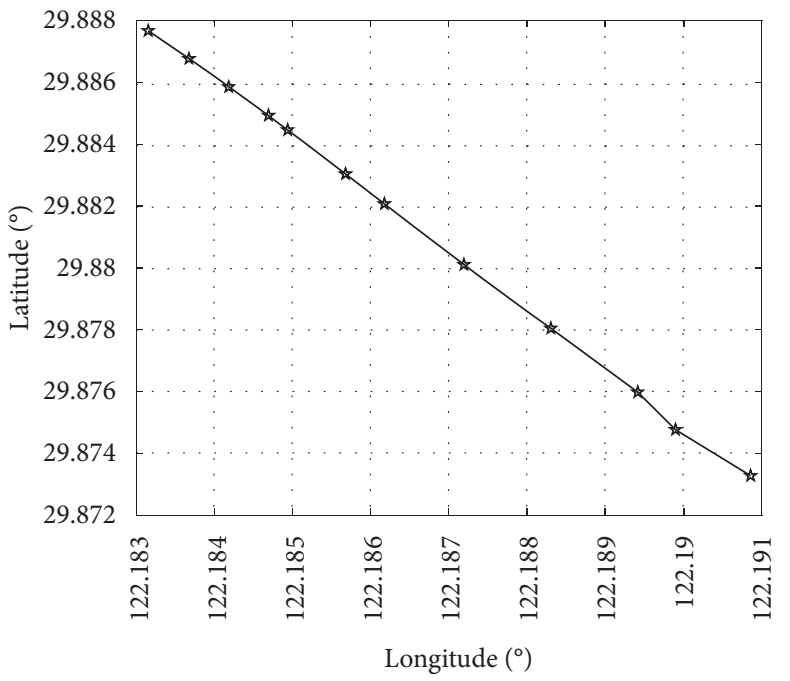

Figure 4: Track of SANTA LAURA. 
TABLE 4: Turning slightly to the left of SOFIE MAERSK.

\begin{tabular}{lcccccc}
\hline Longitude & Latitude & $C$ & $V$ & Head & $a_{\mathrm{c}}$ & $a_{s}$ \\
\hline 122.1663 & 29.915511 & 323.8 & 11.6 & 318.0 & - & - \\
122.165706 & 29.916073 & 319.3 & 11.5 & 316.0 & -3.8 & 0.1 \\
122.164819 & 29.916836 & 315.5 & 11.6 & 315.0 & -2.8 & 0.2 \\
122.164119 & 29.9173685 & 314.6 & 11.7 & 315.0 & -1.7 & 0.1 \\
122.163419 & 29.917901 & 314.0 & 11.7 & 315.0 & -1.4 & 0.1 \\
122.1627195 & 29.9184335 & 312.6 & 11.8 & 314.5 & -3.0 & 0.2 \\
122.16202 & 29.918966 & 311.6 & 11.8 & 314.0 & -1.5 & -0.6 \\
122.159766 & 29.920626 & 311.1 & 11.6 & 304.0 & - & - \\
\hline
\end{tabular}

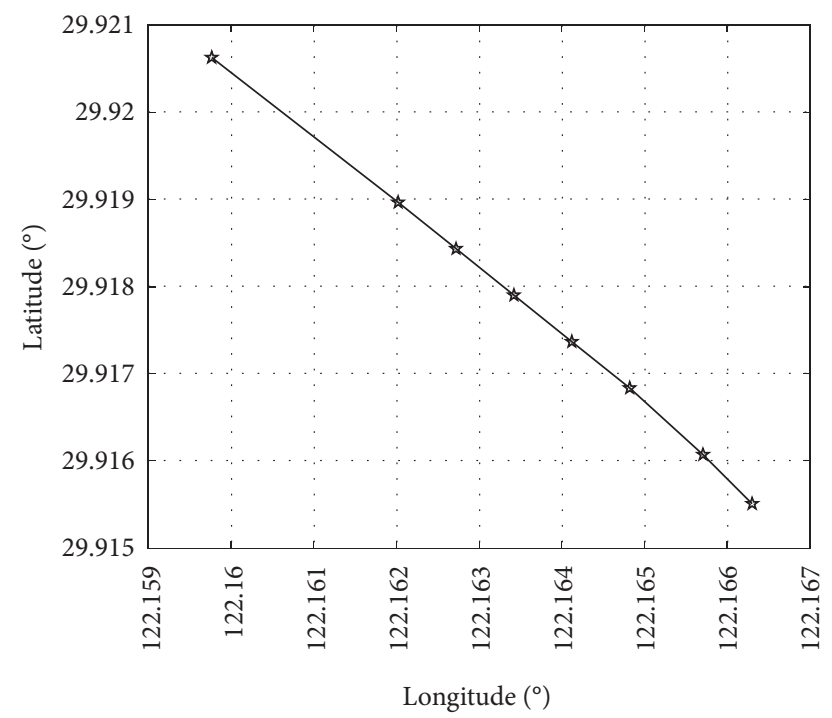

FIgURE 5: Track of SOFIE MAERSK.

TABle 5: Turning slightly to the right of HAIYANG989.

\begin{tabular}{lcccccc}
\hline Longitude & Latitude & $C$ & $V$ & Head & $a_{c}$ & $a_{s}$ \\
\hline 121.992531 & 29.948695 & 122.7 & 7.1 & 159.0 & - & - \\
121.993433 & 29.947961 & 124.8 & 7.3 & 159.0 & 3.9 & 0.3 \\
121.994104 & 29.947551 & 126.1 & 7.4 & 159.0 & 3.6 & 0.3 \\
121.994775 & 29.947156 & 127.3 & 7.5 & 160.0 & 1.6 & 0.0 \\
121.995381 & 29.94673 & 128.9 & 7.6 & 160.0 & 4.7 & 0.1 \\
121.995988 & 29.946305 & 130.4 & 7.6 & 161.0 & 9.3 & 0.0 \\
121.996566 & 29.945835 & 133.5 & 7.6 & 161.0 & - & - \\
\hline
\end{tabular}

4.2.6. Turn Sharply to the Left. Table 6 lists information of KAPITAN AFANASYEV ship, which belongs to turning sharply to the left mode.

The trajectory is plotted in Figure 7.

4.2.7. Turn Sharply to the Right. Table 7 lists information of KAPITAN AFANASYEV ship, which belongs to turning sharply to the right mode.

The trajectory is plotted in Figure 8.

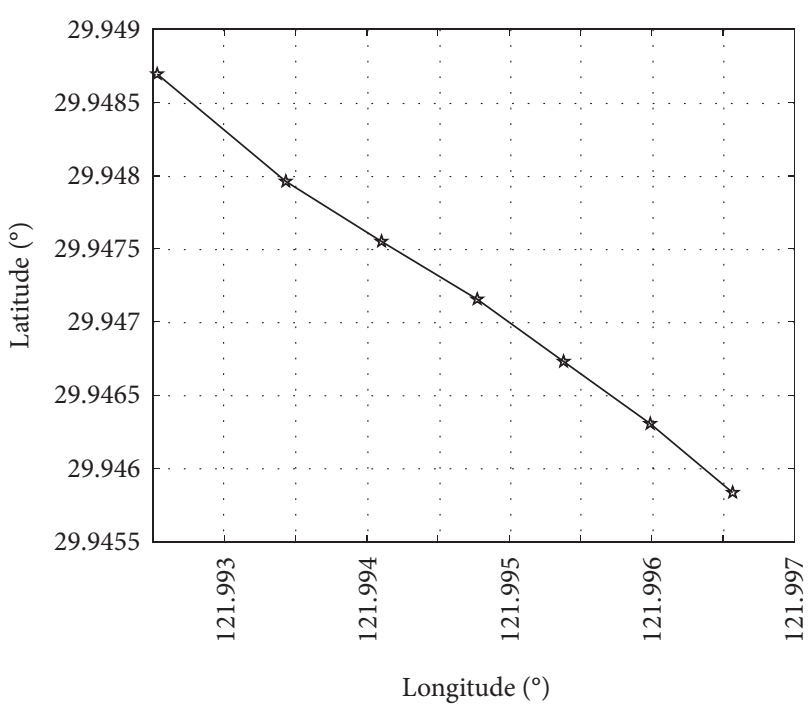

Figure 6: Track of HAIYANG989.

TABLE 6: Turning sharply to the left of KAPITAN AFANASYEV.

\begin{tabular}{lcccccc}
\hline Longitude & Latitude & $C$ & $V$ & Head & $a_{\mathrm{c}}$ & $a_{s}$ \\
\hline 122.1959 & 29.86381 & 342.7 & 9.8 & 345 & - & - \\
122.1956 & 29.86431 & 343.2 & 9.7 & 345 & -7.3 & -0.5 \\
122.1947 & 29.8671 & 335.9 & 9.2 & 327 & -10.5 & -0.6 \\
122.1945 & 29.86737 & 332.4 & 9 & 326 & -18.0 & -0.3 \\
122.1943 & 29.86764 & 326.4 & 8.9 & 325 & -5.6 & 0.0 \\
122.1939 & 29.86806 & 320.8 & 8.9 & 326 & - & - \\
\hline
\end{tabular}

\subsection{Prediction Results}

4.3.1. Results with Different Traffic Busy Degrees. Figure 9 shows the ship track prediction with MMSI 413705970.

Figure 10 shows the longitude prediction error of the ship with MMSI 413705970 on December 30, 2019.

Figure 11 shows its prediction error of latitude with MMSI 413705970.

Figure 12 shows the ship track prediction with MMSI 412600383.

Figure 13 shows its prediction error of longitude.

Figure 14 shows the prediction error of latitude with MMSI 412600383.

The above figures show that the new algorithm can accurately predict the ship track.

4.3.2. Results with Different Fractional Orders. The test and training accuracy with different fractional orders and batch sizes are listed in Table 8. The maximal iteration is 100 .

Table 8 shows that the testing and training error differs with different fractional orders. The maximum training accuracy is obtained when the fractional order is 0.3 . When the fractional order is 0.3 , the test accuracy is the highest.

The test accuracy with different fractional orders and batch sizes is shown in Figure 15. 


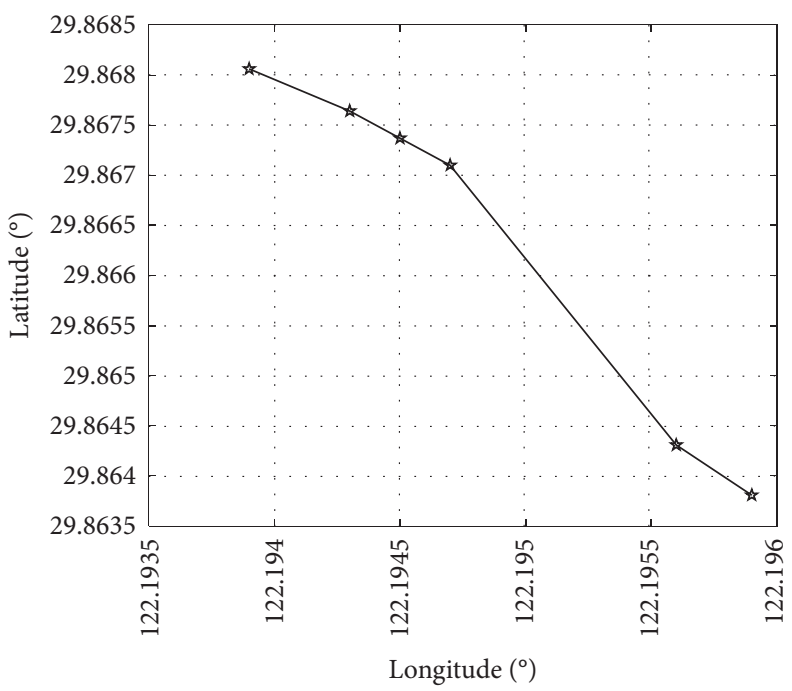

Figure 7: Track of KAPITAN AFANASYEV.

TABLE 7: Turning sharply to the right of KAPITAN AFANASYEV.

\begin{tabular}{|c|c|c|c|c|c|c|}
\hline Longitude & Latitude & $C$ & V & Head & $a_{\mathrm{c}}$ & $a_{s}$ \\
\hline 122.161287 & 29.907758 & 122.9 & 13.9 & 128 & - & - \\
\hline 122.162012 & 29.907304 & 126.1 & 14.0 & 133 & 24.0 & -0.9 \\
\hline 122.16241 & 29.907017 & 134.1 & 13.7 & 135 & 30.0 & 1.2 \\
\hline 122.162679 & 29.906807 & 144.1 & 14.1 & 137 & 15.3 & 0.0 \\
\hline 122.163062 & 29.906494 & 159.5 & 14.1 & 140 & 20.0 & 0.0 \\
\hline 122.16333 & 29.906264 & 166.1 & 14.1 & 141 & 24.0 & -0.6 \\
\hline 122.163753 & 29.905811 & 174.1 & 13.9 & 144 & 60.0 & 0.0 \\
\hline 122.163892 & 29.905654 & 234.1 & 13.9 & 144 & 22.9 & 0.5 \\
\hline 122.164364 & 29.90506 & 241.8 & 14.1 & 145 & 18.0 & 0.0 \\
\hline 122.164396 & 29.905001 & 247.8 & 14.1 & 145 & - & - \\
\hline
\end{tabular}

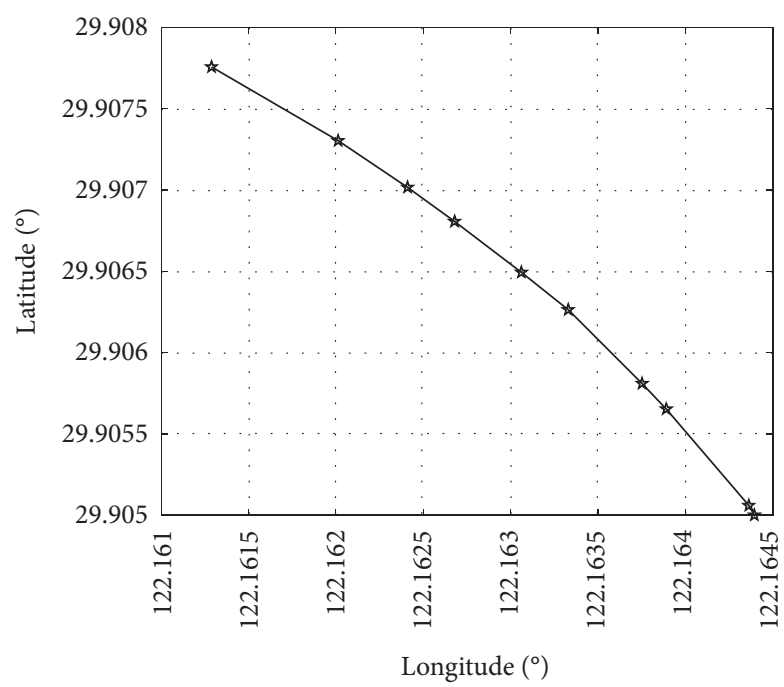

Figure 8: Track of KAPITAN AFANASYEV.

The train accuracy with different fractional orders and batch sizes is shown in Figure 16.

Figure 17 shows the loss function curve.

Figure 18 shows the accuracy curve.

Figure 19 shows the neural network weight curve.

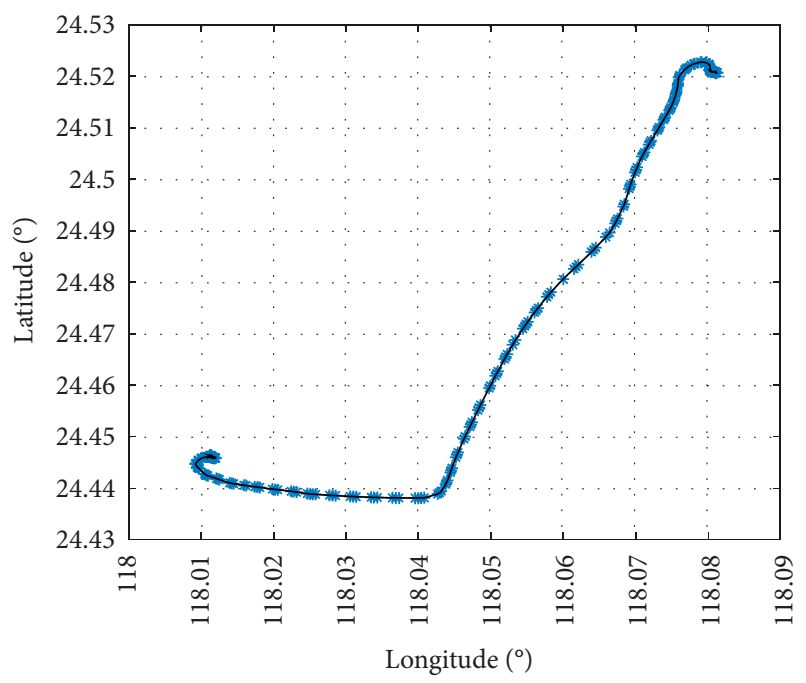

FIGURE 9: Ship track prediction with MMSI 413705970 on December 30, 2019.

4.3.3. Comparison. The proposed algorithm is compared with RNN and FRNN without maneuvering behavior identification (MBI). The errors of longitude of the ship with MMSI 413705970 are shown in Figure 20. 


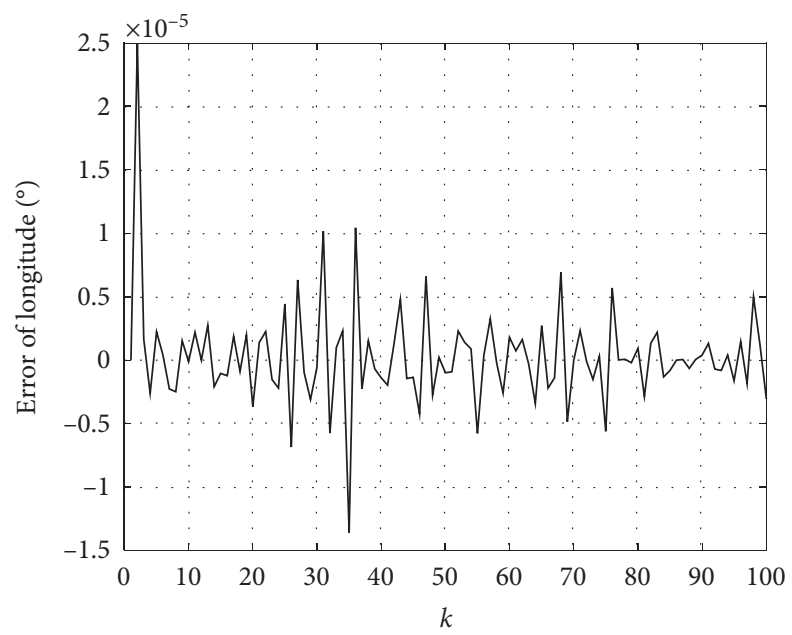

FIGURE 10: Error of longitude with MMSI 413705970.

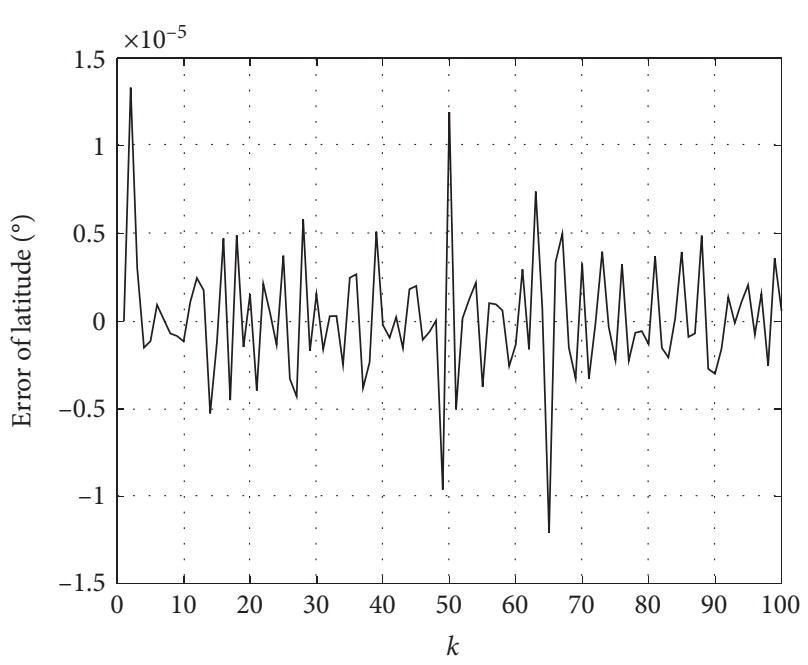

FIGURE 11: Error of latitude with MMSI 413705970.

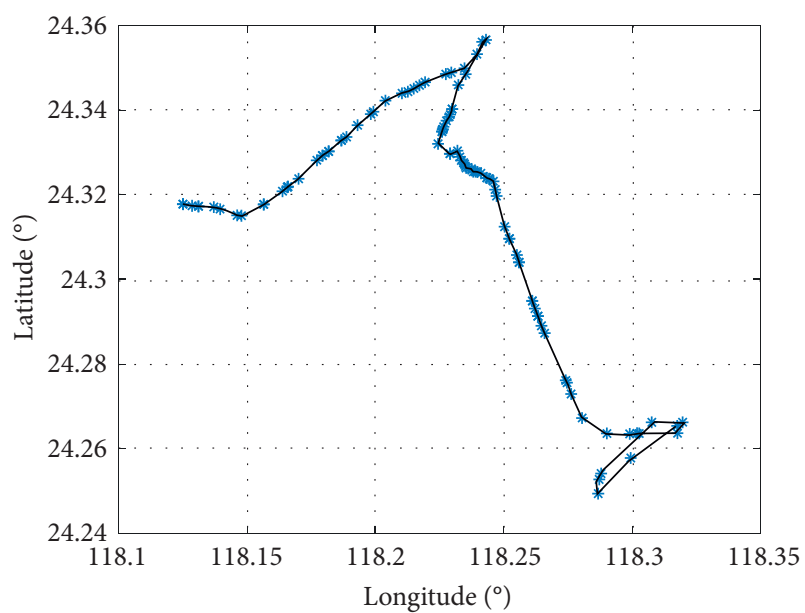

FIgURE 12: Ship track prediction with MMSI 412600383.

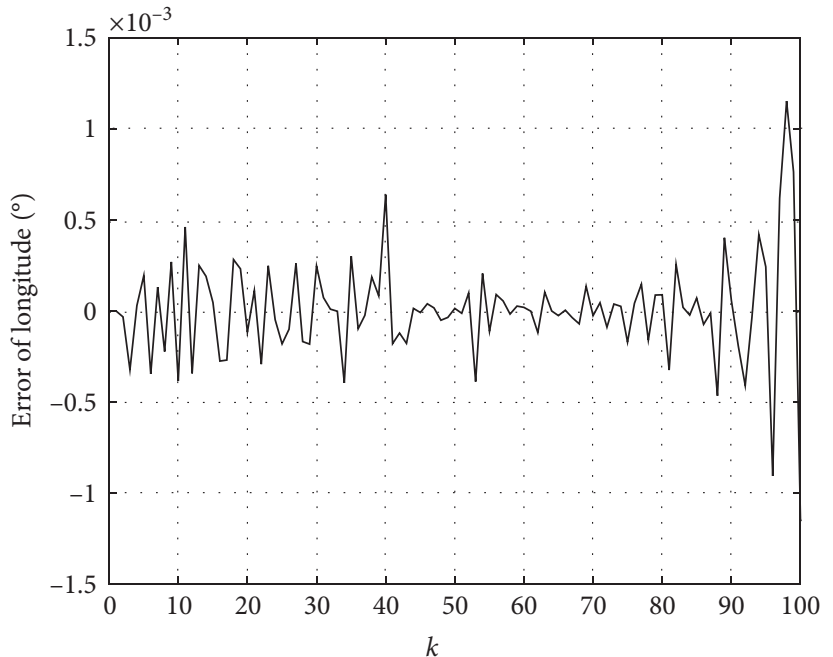

Figure 13: Error of longitude with MMSI 412600383.

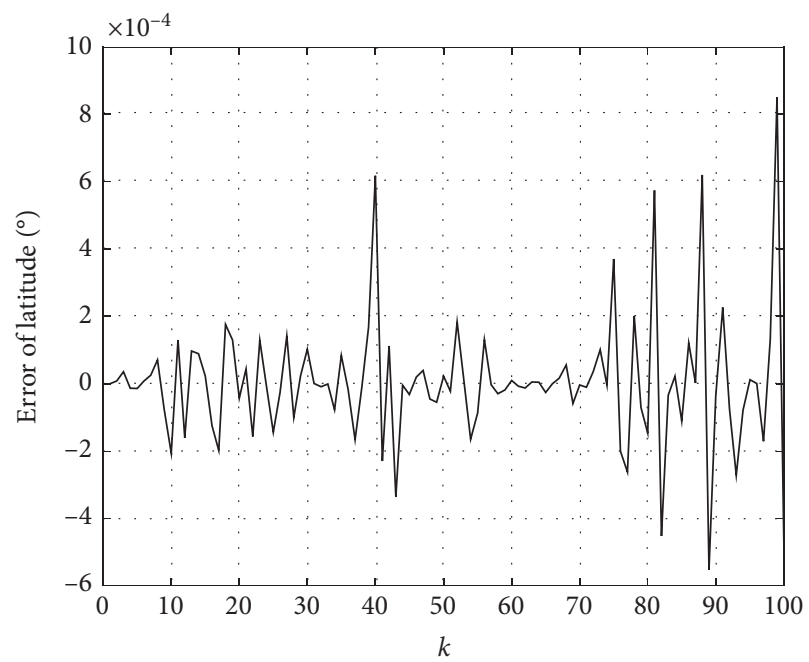

FIgURE 14: Error of latitude with MMSI 412600383. 
TABLE 8: Testing and training accuracy with different fractional orders and batch sizes.

\begin{tabular}{lcccccc}
\hline & & 200 & & 300 & & \\
\hline$\alpha$ & Testing & Training & Testing & Training & Testing & Training \\
0.9 & $94.350000 \%$ & $95.110000 \%$ & $95.590000 \%$ & $96.000000 \%$ & $95.780000 \%$ & $96.478333 \%$ \\
0.8 & $95.100000 \%$ & $95.791667 \%$ & $95.500000 \%$ & $96.326667 \%$ & $95.210000 \%$ & $96.103333 \%$ \\
0.7 & $95.130000 \%$ & $95.683333 \%$ & $95.800000 \%$ & $96.305000 \%$ & $95.750000 \%$ & $96.450000 \%$ \\
0.6 & $95.050000 \%$ & $95.708333 \%$ & $95.660000 \%$ & $96.345000 \%$ & $95.430000 \%$ & $96.188333 \%$ \\
0.5 & $93.550000 \%$ & $94.170000 \%$ & $95.800000 \%$ & $96.385000 \%$ & $95.130000 \%$ & $96.215000 \%$ \\
0.4 & $94.730000 \%$ & $95.641667 \%$ & $95.610000 \%$ & $96.343333 \%$ & $95.670000 \%$ & $96.533333 \%$ \\
0.3 & $95.550000 \%$ & $96.373333 \%$ & $95.810000 \%$ & $96.668333 \%$ & $95.820000 \%$ & $96.696667 \%$ \\
0.2 & $95.280000 \%$ & $96.091667 \%$ & $95.960000 \%$ & $96.720000 \%$ & $96.030000 \%$ & $96.866667 \%$ \\
0.1 & $95.500000 \%$ & $96.278333 \%$ & $95.580000 \%$ & $96.416667 \%$ & $96.050000 \%$ & $96.876667 \%$ \\
\hline
\end{tabular}

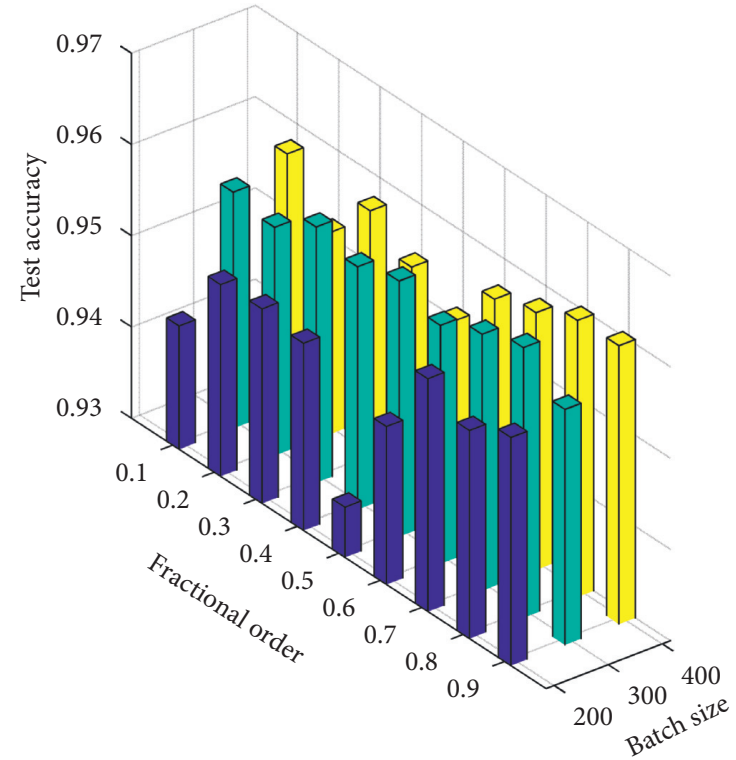

Figure 15: Test accuracy.

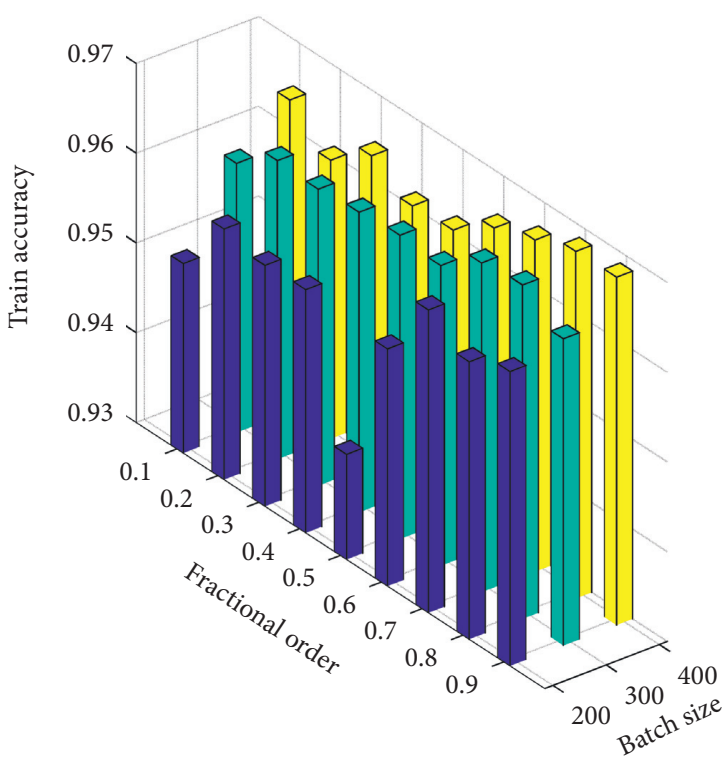

FIgURE 16: Train accuracy.

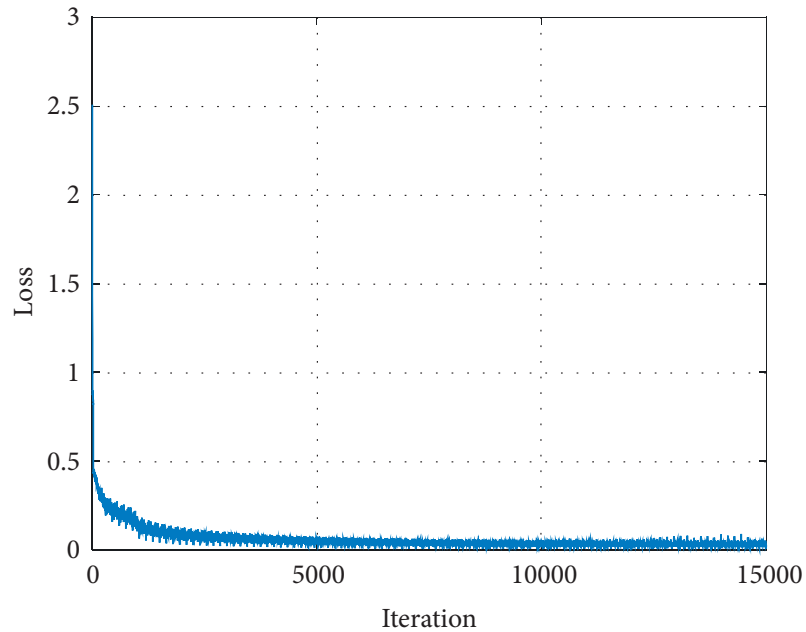

FIgURE 17: Loss function curve.

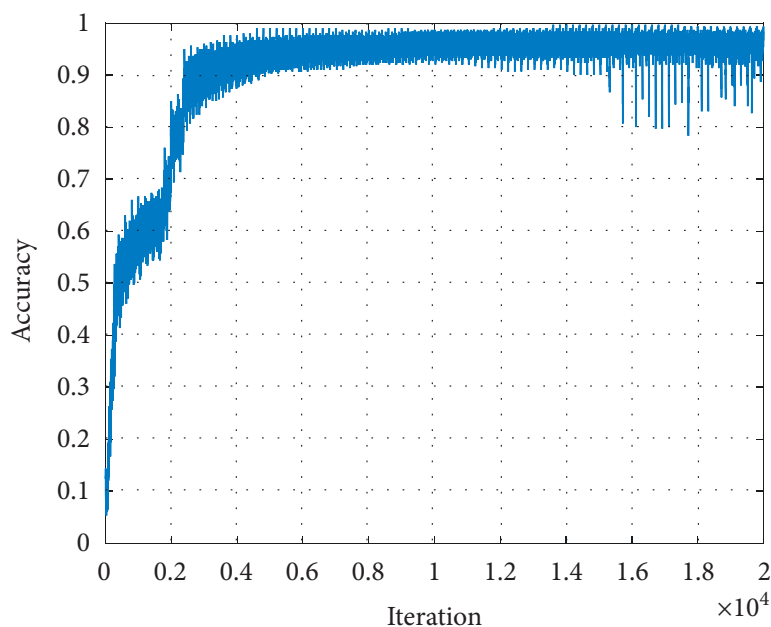

Figure 18: Accuracy curve.

The errors of latitude are shown in Figure 21.

Figures 20 and 21 show that the performance of the proposed algorithms is better than that of RNN and FRNN without maneuvering behavior identification. 


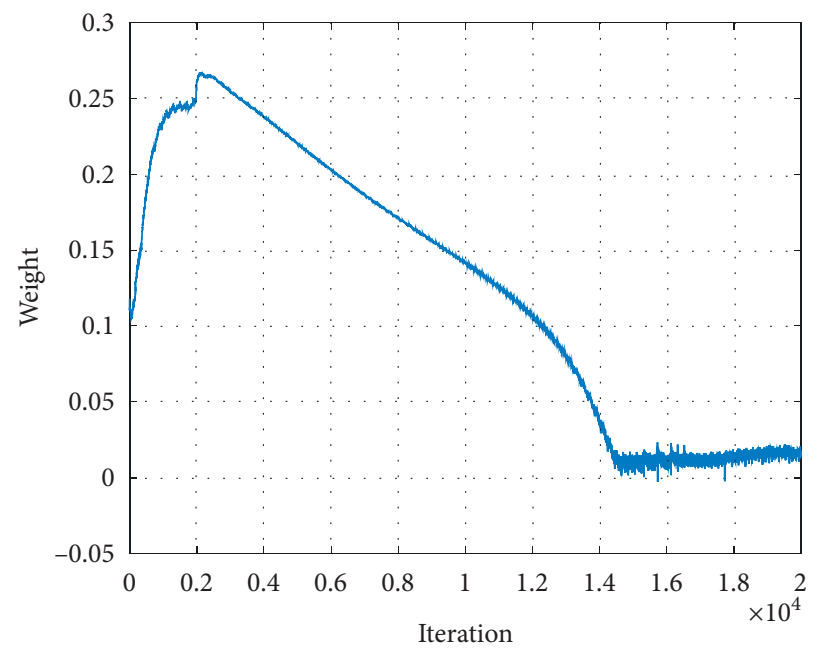

Figure 19: Neural network weight curve.

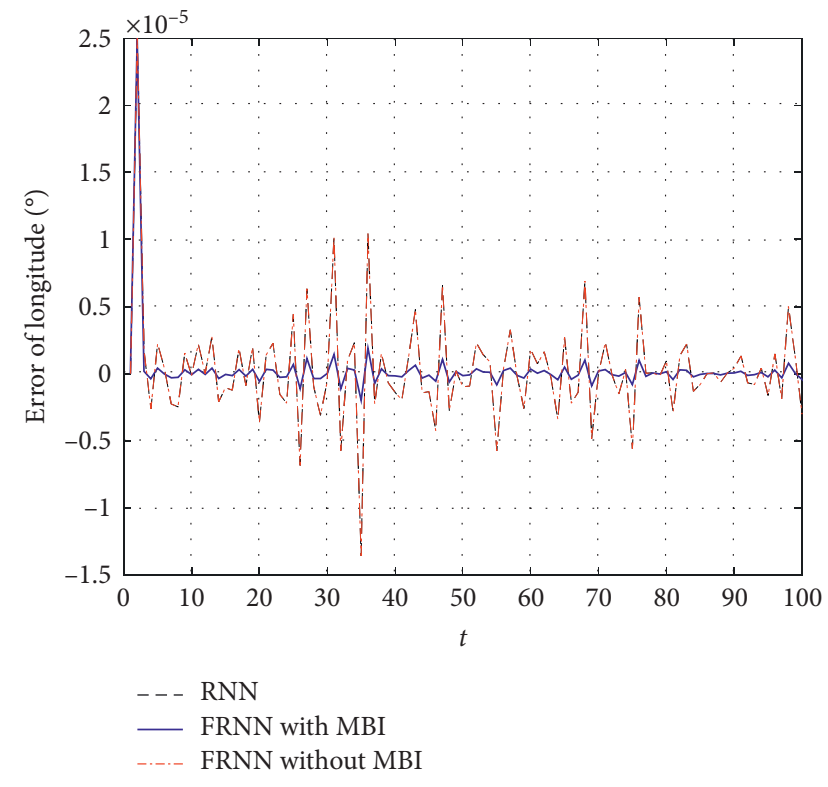

Figure 20: Errors of longitude.

\section{Conclusion}

This paper utilizes fractional gradient descent to improve RNN. The proposed algorithm can adaptively learn the optimal weights. It complies with the literature review compared with RNN and FRNN without maneuvering behavior identification, which can improve prediction accuracy.

The academic implications are applying fractional-order calculus to gradient descent for training recurrent neural network. The convergence of fractional gradient recurrent neural network is proved.

The limitations of the paper are not able to predict long time track. In future studies, the input data of the track prediction model will include the dynamic information of

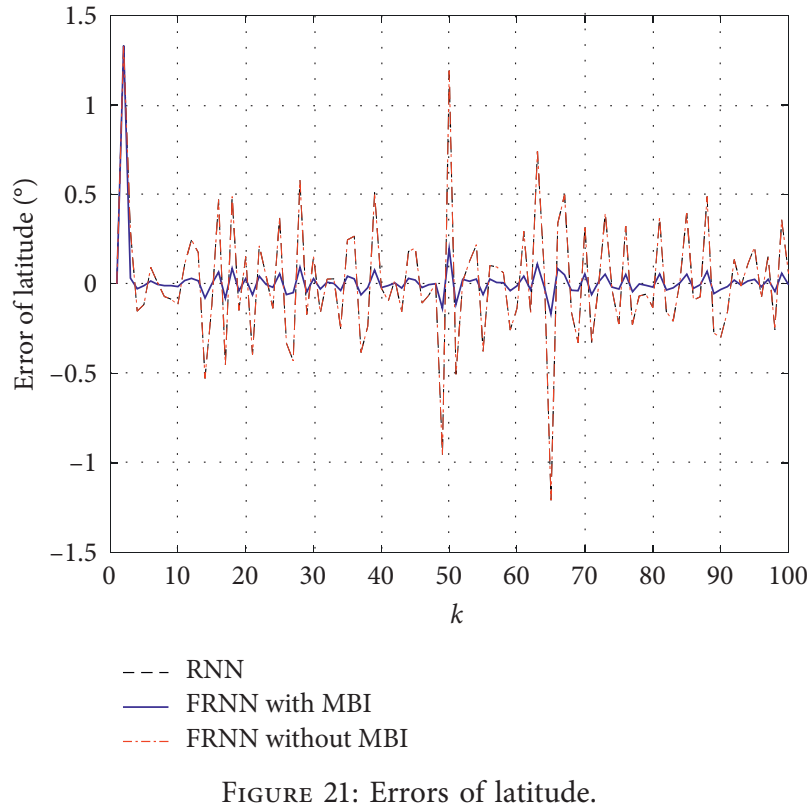

the surrounding ships and the constraint information of the geographical environment, so as to achieve the long-term prediction of the ship's track or under some sudden or extreme conditions. The kinematic model of the ship will be considered. The influence of different time intervals on the ship's trajectory will be measured.

\section{Data Availability}

The data used to support the findings of this study are included within the article.

\section{Conflicts of Interest}

The authors declare that they have no conflicts of interest. 


\section{Acknowledgments}

This work was supported in part by the Natural Science Foundation of Fujian Province (2018J05085), High level research and cultivation fund of transportation engineering discipline of Jimei University (HHXY2020003), and National Natural Science Foundation Cultivation Project of Jimei University (ZP2020005).

\section{References}

[1] Y. Liu, W. Duan, L. Huang, S. Duan, and X. Ma, "The input vector space optimization for LSTM deep learning model in real-time prediction of ship motions," Ocean Engineering, vol. 213, Article ID 107681, 2020.

[2] B. Murray and L. P. Perera, "A dual linear autoencoder approach for vessel trajectory prediction using historical AIS data," Ocean Engineering, vol. 209, Article ID 107478, 2020.

[3] H. Rong, A. P. Teixeira, and C. Guedes Soares, "Ship trajectory uncertainty prediction based on a Gaussian Process model," Ocean Engineering, vol. 182, pp. 499-511, 2019.

[4] J. L. Elman, "Finding structure in time," Cognitive Science, vol. 14, no. 2, pp. 179-211, 1990.

[5] M. Schuster and K. K. Paliwal, "Bidirectional recurrent neural networks," IEEE Transactions on Signal Processing, vol. 45, no. 11, pp. 2673-2681, 1997.

[6] S. Hochreiter and J. Schmidhuber, "Long short-term memory," Neural Computation, vol. 9, no. 8, pp. 1735-1780, 1997.

[7] Y. Chen, "LSTM recurrent neural network prediction algorithm based on Zernike modal coefficients," Optik, vol. 203, Article ID 163796, 2020.

[8] J. Wang, J. Cao, and S. Yuan, "Shear wave velocity prediction based on adaptive particle swarm optimization optimized recurrent neural network," Journal of Petroleum Science and Engineering, vol. 194, Article ID 107466, 2020.

[9] R. R. Borhade and M. S. Nagmode, "Modified atom search optimization-based deep recurrent neural network for epileptic seizure prediction using electroencephalogram signals," Biocybernetics and Biomedical Engineering, vol. 40, no. 4, pp. 1638-1653, 2020.

[10] L.-L. Huang, J. H. Park, G.-C. Wu, and Z.-W. Mo, "Variable-order fractional discrete-time recurrent neural networks," Journal of Computational and Applied Mathematics, vol. 370, Article ID 112633, 2020.

[11] S. Yang, J. Yu, C. Hu, and H. Jiang, "Quasi-projective synchronization of fractional-order complex-valued recurrent neural networks," Neural Networks, vol. 104, pp. 104-113, 2018.

[12] X. Li, J.-A. Fang, W. Zhang, and H. Li, "Finite-time synchronization of fractional-order memristive recurrent neural networks with discontinuous activation functions," Neurocomputing, vol. 316, pp. 284-293, 2018.

[13] Z. Xiao, X. Fu, L. Zhang et al., "Big data driven vessel trajectory and navigating state prediction with adaptive learning, motion modeling and particle filtering techniques," IEEE Transactions on Intelligent Transportation Systems, pp. 1-14, 2020.

[14] R. W. Liu, J. Nie, S. Garg, Z. Xiong, Y. Zhang, and M. S. Hossain, "Data-driven trajectory quality improvement for promoting intelligent vessel traffic services in 6G-enabled maritime IoT systems," IEEE Internet of Things Journal, 2020.

[15] Y. Huang, Y. Li, Z. Zhang, and R. W. Liu, "GPU-accelerated compression and visualization of large-scale vessel trajectories in maritime IoT industries," IEEE Internet of Things Journal, vol. 7, no. 11, pp. 10794-10812, 2020.

[16] C. Luo, H. Fei, D. Sailike, T. Xu, and F. Huang, "Optimization of continuous berth scheduling by taking into account double-line ship mooring," Scientific Programming, vol. 2020, Article ID 8863994, 11 pages, 2020. 